\title{
Polymorphism studies on microRNA targetome of thalassemia
}

\section{Hamid Galehdari1 ${ }^{*}$, Seyedeh Zohreh Azarshin1,2, Mehdi Bijanzadeh¹, Mohammad Shafiei ${ }^{1,2}$}

1Thalassemia \& Hemoglobinopathy Research center, research institute of Health, Ahvaz Jundishapur University of Medical Sciences, Ahvaz, Iran; ${ }^{2}$ Department of Genetics, Faculty of Sciences, Shahid Chamran University of Ahvaz, Ahvaz, Iran; Hamid Galehdari - Email: galehdari187@yahoo.com; *Corresponding author

Received May 3, 2018; Revised May 6, 2018; Accepted May 6, 2018; Published May 31, 2018

$$
\text { doi:10.6026/97320630014252 }
$$

\begin{abstract}
:
Thalassemia is one of the most prevalent hemoglobin disorders. It is caused by the decreased or absent synthesis of one globin chain that leads to moderate to severe hemolytic anemia in clinical complications. Some genetic factors cause these phenotypic variations by the alteration of gene expression. MicroRNAs (miRNAs) are post-transcriptional regulators in gene expression. Therefore, variations in 3'-untranslated region (3'-UTR) of target genes may affect gene expression. It is of interest to evaluate the impact of noncoding SNPs in thalassemia related genes on miRNA: mRNA interactions in the severity of thalassemia. Polymorphisms that alter miRNA: mRNA interactions were predicted using PolymiRTS and Mirsnpscore tools. Then, the effect of predicted target SNPs on thermodynamic stability, local RNA structure and regulatory elements was investigated using RNAhybrid, RNAsnp and RegulomeDB, respectively. The molecular functions and the Biological process of candidate genes were extracted and interaction network was created. Forty-six SNPs were predicted to affect 188 miRNA interactions. These results suggest that 3'-UTR SNP may affect gene expression and cause phenotypic variation in thalassemia patients.
\end{abstract}

Keywords: MicroRNA, polymorphism, thalassemia

\section{Background:}

Thalassemia is one of the most frequent inherited blood disorders with an autosomal recessive inheritance in the world. $\beta$ thalassemia is the result of decreased or complete absence of $\beta$ globin chain expression, leading to the imbalances of $\alpha / \beta$ rate. Excess alpha-chains precipitate in the erythroid cells and result in oxidative damage to the cell membrane, ineffective erythropoiesis and anemia [1]. Thalassemia intermedia are characterized by a wide clinical spectrum from the asymptomatic carrier state to more severe transfusion-dependent thalassemia major. Prediction of clinical severity in $\beta$-thalassemia intermedia patients is difficult due to some genetic factors that have been identified as modifying factors [2]. These factors cause phenotypic diversity, such as severity of anemia, bone marrow hyperplasia, need to blood transfusion and splenectomy requirements [3].

The primary modifying factors are the mild or silent mutations in beta-globin locus. These mutations lead to a smaller imbalance $\alpha / \beta$ globin ratio [4]. The second modifiers are co-inheritance $\alpha$ -

ISSN 0973-2063 (online) 0973-8894 (print)

Bioinformation 14(5): 252-258 (2018) thalassemia or genetic variations that can reduce free alphaglobin chains [4]. The product of some genes may reduce cytotoxic effects of free-alpha globin chains [5]. The third modifiers are variations that occur outside the globin gene cluster. These variations can affect bone, iron and bilirubin metabolism [3]. The free-alpha globin chains and iron overload can produce elevated levels of reactive oxygen species (ROS), resulting in oxidative stress in $\beta$-thalassemia. Oxidative stress leads to major complications but antioxidant enzymes protect cells against oxidative damage, such as catalase and glutathione S-transferases [6, 7]. Moreover, osteoporosis is one of the clinical symptoms of thalassemia that is responsible for morbidity in thalassemia major (TM) patients. The COLIA1 gene plays a major role in osteoporosis and has associated with low BMD in TM patients [8].

The gene expression can be controlled by multiple factors, such as transcription factors, activators, enhancers, repressors and translational factors. Among these factors, microRNAs (miRNAs) are the most important regulatory molecules that have a BIOMEDICAL

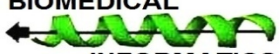
INFORMATICS 
prominent role in post-transcriptional gene regulation. The dysregulation of miRNA may contribute to the pathogenesis of many human diseases, especially hematological disorders [9]. MiRNAs participate in controlling erythropoiesis and globin gene expression. Recent studies have reported that deregulation of miRNAs expression occurs in $\beta$-thalassemia disease. For example, miRNA-451 is up regulated in thalassemic erythroid progenitors. This up-regulation was correlated with disease severity in thalassemia [10]. MiR-486-3p inhibits expression of BCL11A by post-transcriptional mechanism and causes increased synthesis of $\mathrm{HbF}$ in adults [11]. MiRNAs are a class of small $(\sim 22$ nucleotides long), non-coding RNAs. Studies have estimated that $60 \%$ of human protein-coding genes are regulated by miRNAs. Mature single strand miRNA is incorporated into the RNA-induced silencing complex (RISC) and recognizes 3'-untranslated regions (3'-UTRs) of target mRNAs by the Watson-Crick base pairing between seed region of miRNA and target site, leading to repression of mRNA translation or mRNA cleavage [12].

Genetic variations, such as single nucleotide polymorphisms (SNPs) in miRNA binding site of target mRNA may alter thermodynamics energy of RNA duplex and secondary structure of mRNA result in disrupting target site or creating new target site. Therefore, they can affect the regulation of target gene expression [9]. Polymorphisms in miRNA target site may have an association with susceptibility to many human diseases. For example, the rs1044129 within 3'-UTR of the RYR3 gene disrupts a binding site of mir-367 and has been associated with breast cancer risk [13]. The 3'-UTR SNP rs6573 in the PAP1A gene affects the binding site of mir-196a and leads to high expression of PAP1A gene [14]. However, phenotype prediction from genotype is very important for prenatal diagnosis and genetic counseling. To date, Correlation between genotype and phenotype is still unwell understood in TI disorder [15]. MiRNAs and variations in their binding site can moderate the clinical manifestation of diseases. Although, the effect of miRNA-target polymorphism has not been evaluated by both experimental and computational methods in TI disorder till now. The hypothesis of this study, SNPs that located in 3'-UTR of the targeted genes may affect miRNA: mRNA interaction, resulting in alteration of target gene expression and the subsequent clinical symptom of TI disorder. In the present study, SNPs that may alter the strength of miRNA: mRNA duplexes were predicted by in silico procedure. Then, the effective parameters on miRNA: mRNA interactions were evaluated for each SNP. Moreover, the functional role of candidate genes was identified.

\section{Methodology:}

In this study, the genes related to the severity of thalassemia intermedia (TI) were extracted from known literatures [16-20] and the mRNA expression profile and molecular functions of these genes were obtained. Then, miRNA target site SNPs were predicted by in silico analysis. Furthermore, effective parameters in miRNA-target site-SNP (miR-TS-SNP) were analyzed. The workflow of this research was described in Figure 1.

Extraction of gene expression profile:

The expression profile of candidate genes was retrieved from TiGER (Tissue-specific Gene Expression and Regulation) (http:/ / bioinfo.wilmer.jhu.edu/tiger/) to understand the pattern of gene expression and gene regulation in different tissues [21].

\section{MiroRNA target prediction:}

Polymorphism in miRNA target regions can result in the alteration in the miRNA: mRNA interaction. Therefore, the functional SNPs on miRNA target sites in the 3'-UTR of each gene were predicted by using PolymiRTS Database v3.0 (http://compbio.uthsc.edu/miRSN/) [22] and Mirsnpscore (http://www.bigr.medisin.ntnu.no/mirsnpscore/) [23].

\section{Effect of miRNA-target SNPs on miRNA: mRNA hybrid stability:}

The effect of target SNPs on the thermodynamic stability of miRNA: mRNA hybrid was defined with the $\triangle$ MFE $E_{\text {hybrid }}$ score using the RNAhybrid web server (http://bibiserv.techfak.unibielefeld.de/rnahybrid/) [24]. For each interaction, a minimum free energy (MFE) of wild-type (WT) mRNA: miRNA hybrid and alternative (ALT) target mRNA:miRNA hybrid were measured, then the $\triangle \mathrm{MFE}_{\text {hybrid }}$ was computed as $\mathrm{MFE}_{\text {hybrid(alt) }}-\mathrm{MFE}_{\text {hybrid(WT). }}$

Effect of miRNA-target SNPs on the mRNA secondary structures:

The impact of miRNA-target SNPs on the local RNA secondary structure was predicted using the RNAsnp web tool (http://rth.dk/resources/rnasnp/) [25]. The structural difference between WT and variant alleles was calculated by using Euclidean distance or Pearson correlation measure for all sequence intervals. Then, the interval with maximum base pairing distance and the corresponding $p$-value were defined as outputs. Finally, SNPs with $\operatorname{dmax} p$-value $\leq 0.2$ were determined as structure disruptive variants.

Functional annotation of the miRNA-target SNPs:

Functional annotations were analyzed using HaploReg v4.1 (http://www.broadinstitute.org/mammals/haploreg/haploreg. php/) [26] and RegulomeDB (http://regulomedb.org) [27].

\section{Functional enrichment analysis:}

In other to identify the function of candidate genes and affected pathways by SNPs, the biological features were extracted from WebGestalt online tool kit (http://www.webgestalt.org/option.php/) [28] as follows; Gene ontology, gene-phenotype association and gene-disease association. Finally, the gene connection network was imaged using GeneMANIA plugin web app (http://www.genemania.org/plugin/) [29].

\section{Results:}

Most genes are expressed in specific tissues. Therefore, in order to make sure that the candidate genes are expressed in the affected tissues in thalassemia, the expression profile of each gene was obtained from TIGER. All of 26 genes were expressed in at least one of the affected tissues such as blood, bone, bone marrow, heart, liver and spleen (Table 1).

Both PolymiRTS Database v3.0 and Mirsnpscore were used for predicting miRNA:mRNA target SNPs. The retrieved list of miRNA-target SNPs was screened to select modulating SNP. In 
this paper, target-SNP that may reduce the severity of clinical symptoms called modulating SNP. Therefore, SNPs were selected that disrupt miRNA binding sites in antioxidant enzyme genes (CAT, GSTs) and globin synthesis genes (HBB, HBG1 and HBG2). These SNPs may affect gene expression levels, leading to the elevated enzyme activity and the reduced imbalance $\alpha / \beta$ rate, respectively. The selected SNPs for AHSP and HRI genes also disrupt target sites that may result in low levels of alpha-globin, and prevent against the accumulation of alpha globin chains. In contrast, the target-SNPs were selected that create miRNA binding sites in BCL11A, HBA1 and HBA2 genes. These SNPs can lead to the elevated levels of $\mathrm{HbF}$ production and inhibit alphachain synthesis. Finally, 161 modulating SNPs for 415 miRNA were predicted that may affect 438 miRNA: mRNA interactions in 3'-UTR of 20 genes (Supplementary File 1). Then, functional analysis was evaluated for these miRNA-target SNPs. SNPs in 3'UTR region of the gene may create or disrupt the miRNAbinding site. Accordingly, RNAhybrid was used for studying the effect of target SNP on miRNA: mRNA hybrid stability. The negative value of $\triangle \mathrm{MFE}$ demonstrates increase hybrid stability but the positive value of $\triangle \mathrm{MFE}$ indicates the reduction of the hybrid stability. The stability of miRNA-target SNP duplex in 60 out of 438 interactions was reduced and in 25 interactions was increased and 353 miRNA-target SNP interactions were represented no change in the thermodynamic energy by targetSNPs (Figure 2A). RNAsnp was used to predict the effect of target-SNPs on the RNA structural change. Twenty-four targetSNPs out of 161 SNPs have the significant effect on the RNA secondary structure with dmax $p$-value $\leq 0.2$ (Figure 2B, yellow cells in Supplementary File 1).

SNPs in 3'-UTR of genes may affect the binding site of transcription factors. The result from HaploReg was revealed that the motif alteration was occurred in 146 out of 161 SNPs (Supplementary File 1). Moreover, the further analysis was performed using RegulomeDB. Twenty-six SNPs had RegulomeDB score $\leq 3$. Only rs11807 and rs4560 had score 1, likely to alter TF binding and have been associated with gene expression. However, other 24 SNPs had score 2 and 3 with less evidence for the effect on TF binding site (Table 2). Ninety SNPs had score $\geq 4$ with the least binding evidence.

The molecular functions and biological process of candidate genes were identified using WebGestalt (Table 3). The functions, such as antioxidant activity, oxidoreductase activity, peroxidase activity and glutathione transferase activity are involved in detoxification ROS and xenobiotic compounds. Therefore, they can modulate some symptoms of thalassemia. One of the major causes of hemoglobinopathy is disruption of functions, like oxygen binding, heme binding and oxygen transporter activity. The analysis of gene-phenotype association demonstrated an abnormality in the heme biosynthesis and erythrocytes, splenomegaly and anemia. Therefore, expression of the candidate genes in the affected tissues can indicate the major role of these genes in the modulation of thalassemia severity. In addition, the gene network was constructed using the GeneMANIA server, under the biological process weighting. The result of network analysis indicated several networks between the set of candidate genes including, physical interactions, co-localization, co- expression and pathway. It confirms that variations in 3'-UTR region of each gene can affect these networks and phenotype severity related to thalassemia (Figure 3).

\section{Prioritization of the miRNA-target-SNPs:}

The candidate target-SNPs were prioritized which influence on the reduced severity of thalassemia symptoms. Accordingly, the target-SNPs were selected that caused more miRNA-mRNA interactions with $\Delta \leq 0$ and SNPs also altered binding motifs and TFs, and have likely effect on the gene expression. Finally, 46 SNPs were predicted as a potential reducer of thalassemia symptoms (Green cells in Supplementary File 1).

Table 1.The candidate gene expression data in thalassemia uisng Expressed Sequence Tag (EST) Profile from Tiger

\begin{tabular}{ll}
\hline Gene & Tissue \\
\hline HBA1 & Spleen, Thymus, liver, Blood \\
HBA2 & Spleen, Thymus, liver, Blood, Bone_Marrow, \\
HBB & Blood, Bone_Marrow, Muscle, Spleen \\
HBG1 & Spleen, Thymus, Liver \\
HBG2 & Spleen, Thymus, Liver \\
BCL11A & Lymph_Node, Thymus, Blood, Bone_marrow \\
EIF2AK1 & Bladder, Blood, Spleen, Liver, Kidney, Bone, \\
AHSP & Bone_marrow, Testis \\
COL1A1 & Blood, Spleen, Bone_marrow, Heart, Liver \\
CAT & Blood, Bone_marrow, Liver, Spleen \\
GSTA1 & Liver, Testis \\
GSTA2 & Liver, Testis, Kidney, Lung \\
GSTA3 & Placenta, Liver, Testis \\
GSTA4 & Heart, Kidney, PNS, Testis, Brain, Bone, \\
GSTA5 & Bone_marrow \\
GSTM1 & Siver, Kidney, Testis \\
GSTM2 & PNS, Ovary, Brain, Blood, Bone, Heart, Liver \\
GSTP1 & Blood, bone, heart, spleen \\
GSTZ1 & Bone, liver, heart, kidney \\
GSTM3 & Blood, Testis, Liver, Bladder, Heart \\
GSTM4 & Spleen. Stomach, Heart, Kidney, Liver, Blood, \\
GSTM5 & Bladder, Bone \\
GSTT1 & Liver, heart, Bladder, blood, Kidney, Bone, Thymus \\
GSTT2 & Thymus, Heart, Liver \\
GSTO1 & Spleen, Heart, Liver, Kidney, Bone, Skin \\
GSTK1 & Blood, Bone, Bone_marrow, Heart, Liver, Spleen, \\
& Testis, Thymus \\
\hline &
\end{tabular}

Table 2.List of miRNA target-SNPs in the binding of transcription factor

\begin{tabular}{lll}
\hline Gene & SNP ID & RegulomeDB score $*$ \\
\hline HBA1 & rs3180978 & 2b \\
HBA2 & rs4021968 & $2 \mathrm{~b}$ \\
& rs4021969 & $2 \mathrm{~b}$ \\
& rs3209669 & $2 \mathrm{~b}$ \\
& rs4021965 & $2 \mathrm{~b}$ \\
& rs3209698 & $2 \mathrm{~b}$ \\
& rs4021970 & $3 \mathrm{a}$ \\
COL1A1 & rs1061947 & $2 \mathrm{a}$ \\
& rs12937105 & $2 \mathrm{~b}$ \\
\hline
\end{tabular}




\begin{tabular}{lll}
\hline & rs12944834 & $2 \mathrm{~b}$ \\
& rs75713851 & $2 \mathrm{~b}$ \\
& rs75168103 & $2 \mathrm{~b}$ \\
& rs11556514 & $2 \mathrm{~b}$ \\
& rs201085309 & $2 \mathrm{~b}$ \\
& rs202170631 & $2 \mathrm{~b}$ \\
& rs19806909 & $2 \mathrm{~b}$ \\
HBG2 & rs34162544 & $3 \mathrm{a}$ \\
HRI & rs200786947 & $3 \mathrm{a}$ \\
& rs3801032 & $2 \mathrm{c}$ \\
GSTM5 & rs4560 & $1 \mathrm{f}$ \\
& rs11807 & $1 \mathrm{~d}$ \\
& rs112412754 & $2 \mathrm{~b}$ \\
& rs116803889 & $2 \mathrm{~b}$ \\
GSTP1 & rs191030500 & $3 \mathrm{a}$ \\
& rs5031031 & $3 \mathrm{a}$ \\
& rs201925035 & $3 \mathrm{a}$ \\
\hline
\end{tabular}

*It represents confidence for a variation to be resided in a functional location. This score was evaluated by supporting data as follow:

1d: eQTL + TF ((transcription factor)) binding + any motif + DNase peak; 1f: eQTL + TF binding / DNase peak; 2b: TF binding + any motif + DNase Footprint + DNase peak; $2 \mathrm{c}$ : TF binding + matched TF motif + DNase peak; 3a: TF binding + any motif + DNase peak.

Table 3: Functional Enrichment annotation od candidate genes

\begin{tabular}{lll}
\hline Gene & SNP ID & RegulomeDB score* \\
\hline HBA1 & rs3180978 & $2 \mathrm{~b}$ \\
HBA2 & rs4021968 & $2 \mathrm{~b}$ \\
& rs4021969 & $2 \mathrm{~b}$ \\
& rs3209669 & $2 \mathrm{~b}$ \\
& rs4021965 & $2 \mathrm{~b}$ \\
& rs3209698 & $2 \mathrm{~b}$ \\
COL1A1 & rs4021970 & $3 \mathrm{a}$ \\
& rs1061947 & $2 \mathrm{a}$ \\
& rs12937105 & $2 \mathrm{~b}$ \\
& rs12944834 & $2 \mathrm{~b}$ \\
& rs75713851 & $2 \mathrm{~b}$ \\
& rs75168103 & $2 \mathrm{~b}$ \\
& rs11556514 & $2 \mathrm{~b}$ \\
& rs201085309 & $2 \mathrm{~b}$ \\
& rs202170631 & $2 \mathrm{~b}$ \\
& rs19806909 & $2 \mathrm{~b}$ \\
HBG2 & rs34162544 & $3 \mathrm{a}$ \\
HRI & rs200786947 & $3 \mathrm{a}$ \\
& rs3801032 & $2 \mathrm{c}$ \\
GSTM5 & rs4560 & $1 \mathrm{f}$ \\
& rs11807 & $1 \mathrm{~d}$ \\
& rs112412754 & $2 \mathrm{~b}$ \\
& rs116803889 & $2 \mathrm{~b}$ \\
GSTP1 & rs191030500 & $3 \mathrm{a}$ \\
& rs5031031 & $3 \mathrm{a}$ \\
& rs201925035 & $3 \mathrm{a}$ \\
\hline
\end{tabular}

aGene ontology; bRate of enrichment was retrieved from the WebGestalt online tool kit database, based on analyzing the ranked gene list and interactions among genes.

\section{Discussion:}

Thalassemia is the hemolytic disorder with phenotypic heterogeneity and causes a wide range of symptoms [1]. Moreover, the understanding of the phenotype-genotype correlation is difficult due to modifying factors in thalassemia [2]. Several studies have been demonstrated that the occurring variation in some genes, such as $H B A 1, H B A 2, H B B, H B G 1$, $H B G 2, B C L 11 A, C O L 1 A 1, C A T$ and GST genes can moderate the severity of thalassemia [16-20]. Recently, several studies have evaluated the effect of 3'-UTR SNPs on gene expression. These studies indicated an important role of miRNA-target-SNP on disease susceptibility $[9,30]$. Furthermore, the computational approaches are useful for the prioritization of functional miRNA target-SNPs in biomedical research. The results of bioinformatics analysis predict probabilistic and potential candidate SNPs for further experimental approaches. Accordingly, this paper was evaluated by the hypothesis that 3'-UTR SNPs in thalassemia relevant genes may affect miRNA regulation and create phenotype diversity. Therefore, the bioinformatics approaches were used to identify functional 3'-UTR SNPs which reduce clinical severity of thalassemia.

First, the gene expression profile indicated all candidate genes have the expression in affected tissue in thalassemia such as, blood, spleen, heart and liver that it confirms variations in candidate genes may influence on the observed phenotype severity. Besides, these genes have an important role in molecular functions, such as antioxidant activity, oxidoreductase activity, peroxidase activity and glutathione transferase activity which are responsible for the protection against free radicals of oxygen, iron overload and reduce sensitivity to oxidative stress. The analysis of gene-phenotype association also indicates an abnormality in the heme biosynthesis and erythrocytes, splenomegaly and anemia by the disrupted candidate genes. List of miRNA targetSNPs was extracted using PolymiRTS and Mirsnpscore tools for each gene. Then, 161 modulating SNPs were selected from this list. In the next step, further analysis was performed: miRNAmRNA hybrid stability, annotation of TF and regulatory element. Twenty-six SNPs have RegulomeDB score $\leq 3$ with likely effect on transcription factor binding. Only two SNPs rs11708 and rs4560 were found with the RegulomeDB score $1 \mathrm{~d}$ and 1f, respectively and associated with gene expression. For example, the rs11807 in the GSTM5 gene was shown association with diagnosis age of Parkinson disease [31]. Another study indicated that SNP rs4560 could regulate gene expression in the lymphoblastoid cell [32]. However, the effect of variations on the local RNA secondary structure was investigated. Finally, out of 161 modulating SNPs, 55 SNPs of them were prioritized as high functional SNPs. Nine SNPs (rs4021970, rs12937105, rs75713851, rs4560, rs1050032, rs405729, rs1055259, rs145451237 and rs191030500) out of 55 functional SNPs were found with RNAsnp $p$-value $\leq 0.2$ which can lead to disruption of RNA secondary structure and create a change in miRNA binding. Hence, there is a limitation for the understanding impact of the altered mRNA structure on how expression level of gene alters in this study. For this purpose, need to further experimental studies for predicting an apparent phenotype by these variations. For example, a recent study has shown that variation in pri-mir-30c-1 sequence can lead to alteration of miRNA secondary structure. This structural 


\section{BIOINFORMATION}

Discovery at the interface of physical and biological sciences

change promotes binding affinity of SR protein and increases expression of the mature miR-30c in breast cancer [33]. Another study had predicted rs75713851, which may have an effect on miRNA binding site and function of lung cancer-related genes [34]. Therefore, the disrupted structural SNPs were ignored from the prioritized functional SNPs. These results lead to the prioritization of $463^{\prime}$-UTR SNPs as modifying factors and 188 miRNA: mRNA interactions, which cause phenotype severity.
Moreover, the findings are helpful to the understanding of the underlying mechanisms that miRNA-target SNPs regulate miRNA and alter gene expression. Moreover, these results contribute to genetic counseling for prenatal diagnosis and effective treatment of patients with thalassemia. However, further experimental validations and association studies should be performed to confirm the importance of these results.

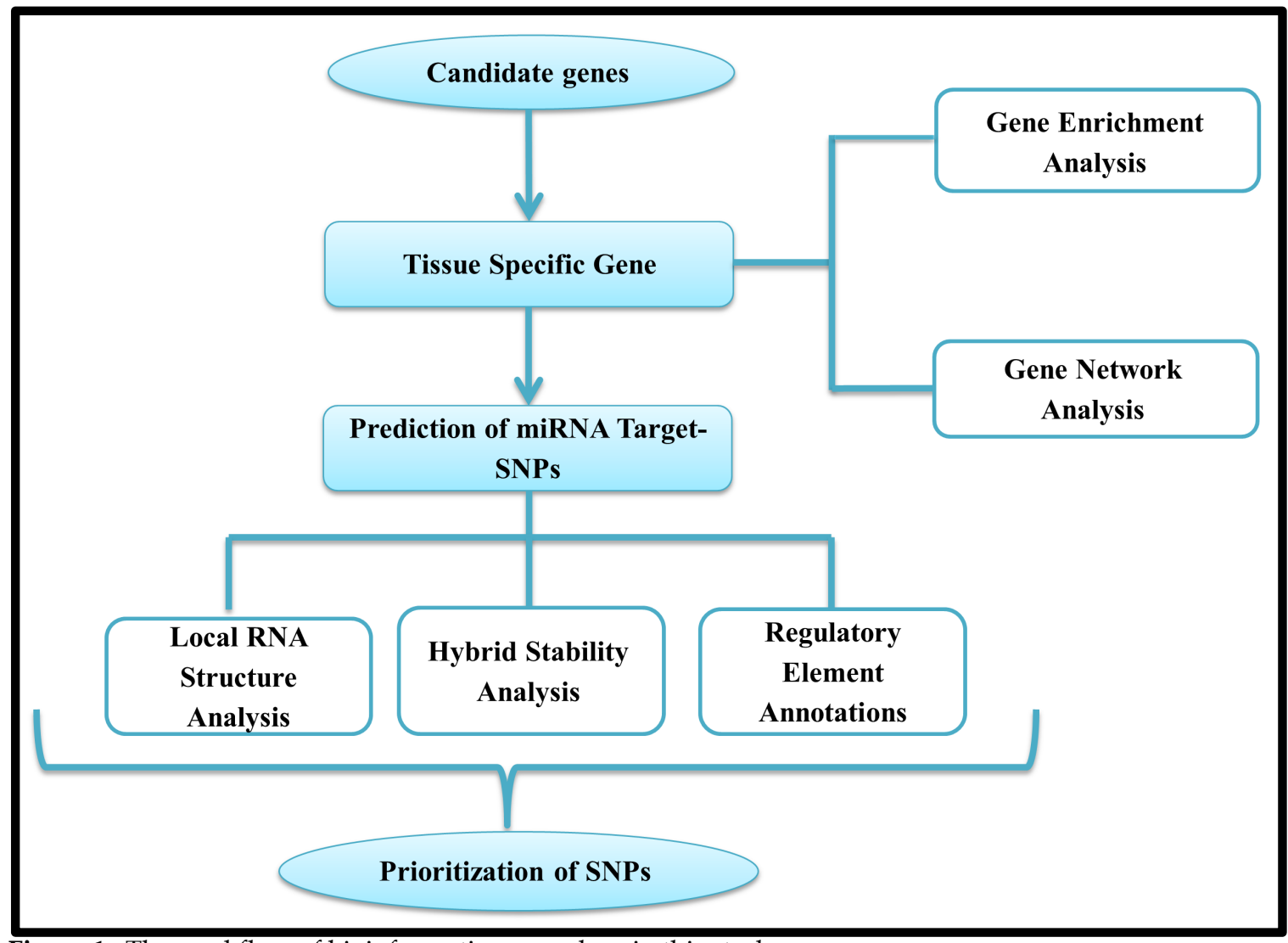

Figure 1. The workflow of bioinformatics procedure in this study

A

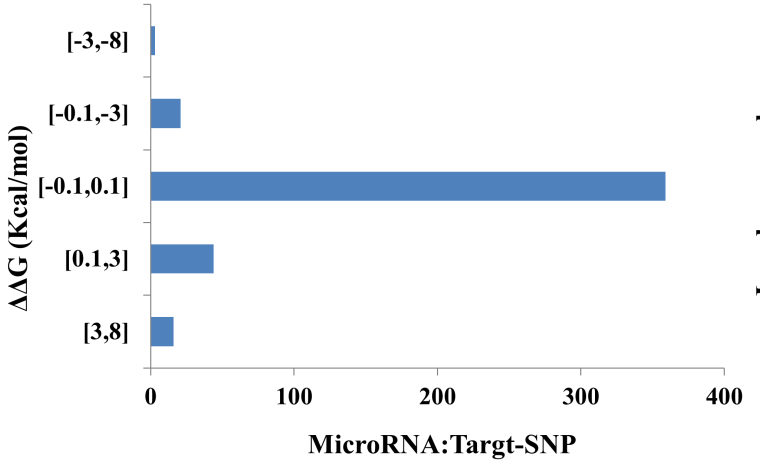

B

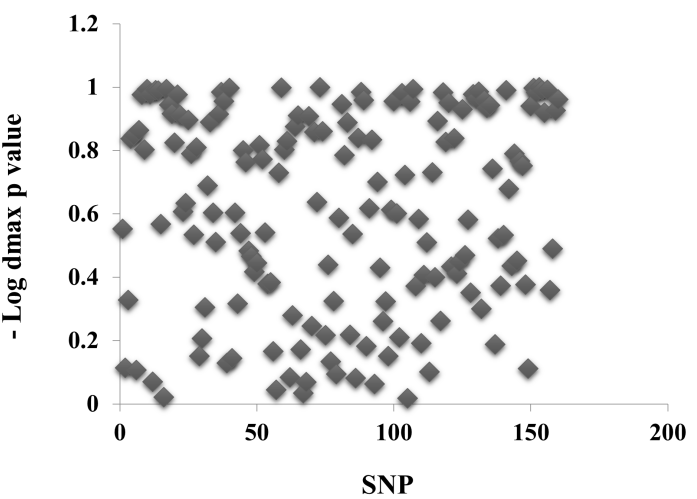

Figure 2: (A) The plot of $\triangle \mathrm{MFE}$ hybrid for miRNA:mRNA (target SNPs) interactions. (B) The distribution plot of dmax $p$-value for targetSNPs by RNAsnp web tool. Each SNP with dmax $P$-value $<0.2$ is significant. 


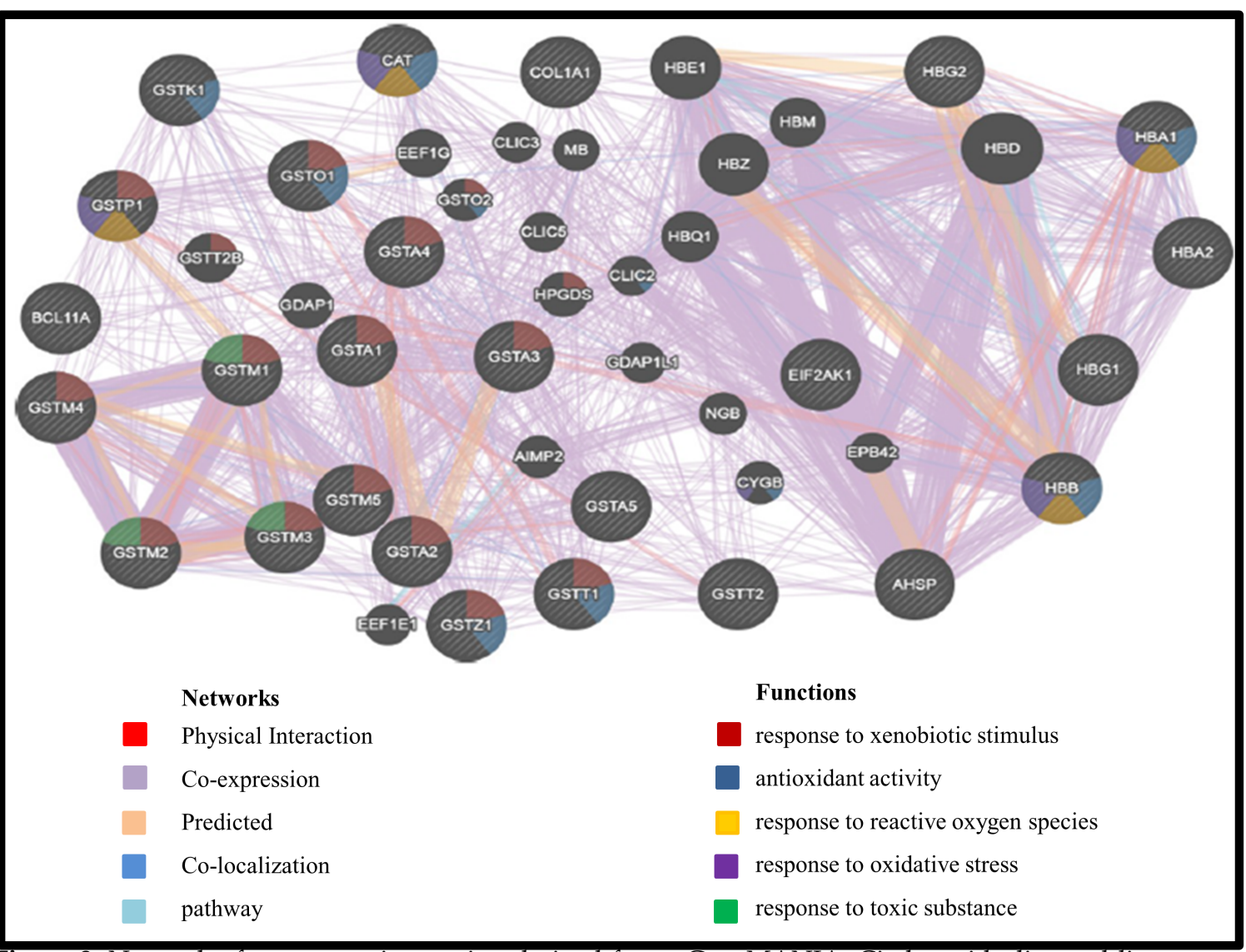

Figure 3. Network of gene-gene interaction derived from GeneMANIA. Circles with diagonal line represent the queried genes and circles without diagonal line represent mediated genes for interactions. These candidate genes represent co-expression, co-localization, predicted and physical interaction networks with themselves. some important functions of genes are shown with the color triangles in the circle: red triangle response to xenobiotic stimulus, blue triangle antioxidant activity, yellow triangle response to reactive oxygen species, violet triangle response to oxidative stress and green triangle response to toxic substance.

\section{Conclusion:}

We report the predicted functional miRNA-target-SNPs. Results suggest that variations in miRNAtarget sites in thalassemia related genes might moderate phenotype severity of thalassemia. These non-coding SNPs can act as modifying factors to reduce $\alpha / \beta$ imbalance rate and cellular oxidative stress. Moreover, these modifiers contribute to the genetic counseling but more experimental studies need to indicate validation of these results.

\section{Acknowledgments:}

We thank Shahid Chamran University of Ahvaz for supporting this study.

\section{References}

[1] Musharraf SG et al. Sci Rep. 20177. [PMID: 28198811].

[2] Nuntakarn L et al. Blood Cells, Mol Dis. 2009 42:1. [PMID:18951049]

[3] Musallam KM et al. Cold Spring Harb Perspect Med. 2012 2:7. [PMID: 22762026]

[4] Taher AT et al. Mediterr J Hematol Infect Dis. 20091:1. [PMID: 21415986]

[5] Surapolchai P et al. Ann Hematol. 2017 96:6. [PMID: 28337528]
[6] Nagy T et al. Arch Biochem Biophys. 2012 525:2. [PMID: 22286031]

[7] Nebert DW \& Vasiliou V.Hum Genomics. 2004 1:6. [PMID: 15607001]

[8] Singh K et al. Gene Ther Mol Biol. 2013 15:1. [PMID: 21178584]

[9] Ghaedi H et al. Genomics. 2015 106:6. [PMID: 26520014]

[10] Svasti S et al. Ann Hematol. 2010 89:10. [PMID: 20461517]

[11] Lulli Vet al. PLoS One. 2013 8: 4. [PMID: 23593217]

[12] Akhtar MM et al. Nucleic Acids Res. 2016 44:1. [PMID: 26578605]

[13] Zhang L et al. Proc Natl Acad Sci. 2011 108:33. [PMID: 21810988]

[14] Wang K et al. Carcinogenesis. 2012 33:11. [PMID: 22859270]

[15] Weatherall DJ. Nat Rev Genet. 2001 2:4. [PMID: 11283697]

[16] Muanprasat C et al. PLoS One. 2013 8:1. [PMID: 23383265]

[17] Sengsuk C et al. Indian J Clin Biochem. 2014 29: 3. [PMID: 24966477]

[18] Uda M et al. Proc Natl Acad Sci. 2008 105:5. [PMID: 26375006]

[19] Han AP et al. J Clin Invest. 2005 115:6. [PMID: 15931390]

[20] Mohammdai-Asl J et al. Biochem Genet. 2015 53:9-10. [PMID: 26143597] 


\section{BIOINFORMATION}

\section{Discovery at the interface of physical and biological sciences}

\section{Open access}

[21] Liu X et al. BMC Bioinformatics. 2008 9:1. [PMID: 18541026]

[22] Bhattacharya A et al. Nucleic Acids Res. 2014 42:D1. [PMID: 28381629]

[23] Thomas LF et al. Nucleic Acids Res. 2011 39:16. [PMID: 21693556]

[24] Kruger J \& Rehmsmeier M. Nucleic Acids Res. 2006 34:Web Server issue. [PMID: 16845047]

[25] Sabarinathan R et al. Nucleic Acids Res. 2013 41:Web Server issue. [PMID: 23630321]

[26] Ward LD \& Kellis M. Nucleic Acids Res. 2016 44:D1. [PMID: 26657631]
[27] Boyle AP et al. Genome Res. 2012 22:9. [PMID: 22955989]

[28] Wang J et al. Nucleic Acids Res. 2013 41:Web Server issue. [PMID: 23703215]

[29] Montojo J et al. F1000Research. 2014 3:153. [PMID: 25254104]

[30] Moszynska A et al. Open Biol. 2017 7:4. [PMID: 28381629]

[31] Searles Nielsen S et al. Int J Mol Epidemiol Genet. 2013 4:1. [PMID: 23565323]

[32] Stranger BE et al. Nat Genet. 2007 39:10. [PMID: 17873874]

[33] Fernandez N et al. Nat Commun. 2017 8. [PMID: 28466845]

[34] He F et al. Asian Pac J Cancer Prev. 2014 15:8. [PMID: 24870764]

Edited by $P$ Kangueane

Citation: Galehdari et al. Bioinformation 14(5): 252-258 (2018) License statement: This is an Open Access article which permits unrestricted use, distribution, and reproduction in any medium, provided the original work is properly credited. This is distributed under the terms of the Creative Commons Attribution License 